\title{
Quantitative Determination of the Nucleophilicity of Allylsilanes
}

\author{
Herbert Mayr* and Gisela Hagen \\ Institut für Chemie, Medizinische Universität zu Lübeck, Ratzeburger Allee 160, D-2400 Lübeck 1, Federal Republic of \\ Germany
}

Kinetic investigations on the reactivity of allylsilanes (1a-0) towards the $p$-methoxy substituted diphenylcarbenium ion (2) are reported.

In spite of the wide use of allylsilanes in organic synthesis, 1 little quantitative information concerning the activation of $\mathrm{C}=\mathrm{C} \pi$-bonds by allylic silyl groups is available. Competition experiments have shown that the reactivity of the $\mathrm{C}=\mathrm{C}$ double bond towards the diphenylmethyl cation increases by 30700 when an allylic hydrogen of propene is replaced by a trimethylsilyl group. ${ }^{2}$ We have now determined the rate constants for the reactions of the allylsilanes $(\mathbf{1 a - 0})$ towards the $p$-anisylphenylcarbenium ion (2) using the kinetic method previously described. ${ }^{3}$

When allyltrimethylsilane (1e) was added to a solution of (2) $-\mathrm{BCl}_{4}-$ in dichloromethane at $-70^{\circ} \mathrm{C}$, the decrease of the carbenium ion concentration, monitored photometrically and conductimetrically, followed a second order rate law [first order with respect to each (1e) and (2)]. ${ }^{3}$ Addition of a tetra-alkylammonium tetrachloroborate or replacement of $\mathrm{BCl}_{4}-$ by $\mathrm{BCl}_{3} \mathrm{OMe}^{-}$or $\mathrm{SnCl}_{5}$ - did not influence the rate of the overall reaction, indicating that the $\beta$-silyl substituted carbenium ion (3) is generated in the rate determining step.

Table 1 shows that the reactivity of allylsilanes strongly depends on the nature of the substituents X and Y. Replacement of methyl by larger alkyl groups (branched or unbranched) leads to a slight increase of reactivity, and exchange of the three methyl groups by phenyl reduces the reactivity by two orders of magnitude. A reduction of reactivity by three orders of magnitude is observed when one methyl group of (1e) is replaced by chlorine (1b). The inductively withdrawing effect of chlorine is cumulated in allyltrichlorosilane (1a). This compound, in contrast to propene, does not react with (2)- $\mathrm{BCl}_{4}-$ at $-70^{\circ} \mathrm{C}$, indicating a deactivation of the double bond by the trichlorosilyl group.

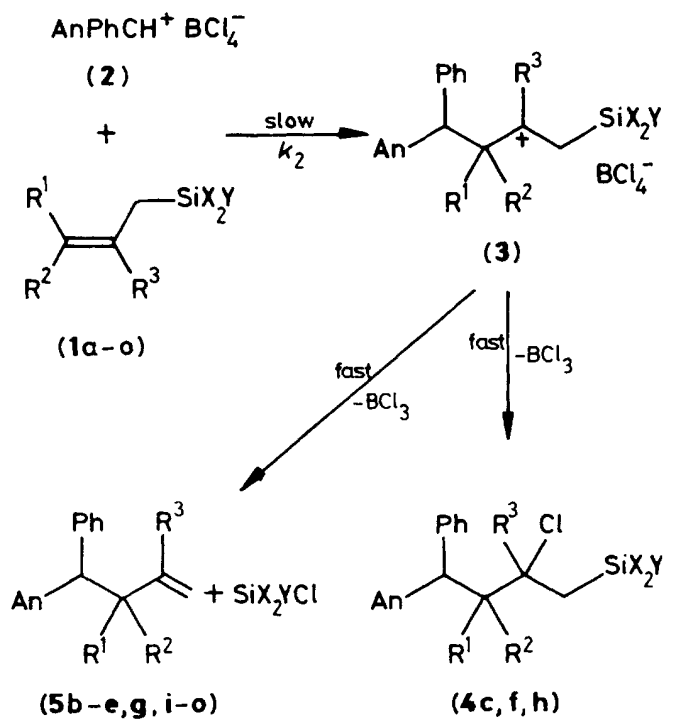

An $=p$-Anisyl
As reported for alkenes, ${ }^{4}$ an additional methyl group at the developing carbenium centre causes a strong reactivity increase, and the addition rate of (1m) is too high to be determined by our method. A quantitative value for the $\alpha$-methyl effect (5950) is derived from the comparison (1c/11). In contrast to (1a), the trichlorosilyl derivative (1k) does react with (2), and the comparison (1k)/isobutene shows a deactivating effect of 360 for the trichlorosilyl group.

One methyl group at the initially attacked vinylic carbon increases the reactivity to a similar degree $[(\mathbf{l n} / \mathbf{1 e})=21]$ as reported for alkenes (trimethylethylene/isobutene $=30$ to 50), ${ }^{4}$ and the expected electronic acceleration by the second methyl group is overcompensated by steric retardation, resulting in a reduced reactivity of (10) compared with (1n).

In summary, the rate effects caused by $\alpha$ - and $\beta$-methyl groups in allylsilanes closely resemble those reported for the corresponding reactions of normal alkenes, an indication for similar transition state structures. ${ }^{5}$ The reactivity scale in Table 1, which refers to the reference electrophile (2) will certainly experience changes when other electrophiles are considered. ${ }^{6}$ We expect, however, only relatively small modifications for other carbon electrophiles if systems with strong steric interactions are excluded, so that the $k$ values in Table 1 should be useful for designing $\mathrm{C}-\mathrm{C}$ bond forming reactions via electrophilic attack at allylsilanes.

Table 1. Second order rate constants for the reactions of the allylsilanes $(\mathbf{1 a - 0})$ with $p$-anisylphenylcarbenium tetrachloroborate (2) $-\mathrm{BCl}_{4}^{-}\left(\mathrm{CH}_{2} \mathrm{Cl}_{2},-70^{\circ} \mathrm{C}, 1 \mathrm{~mol}^{-1} \mathrm{~s}^{-1}\right)$.

$k$

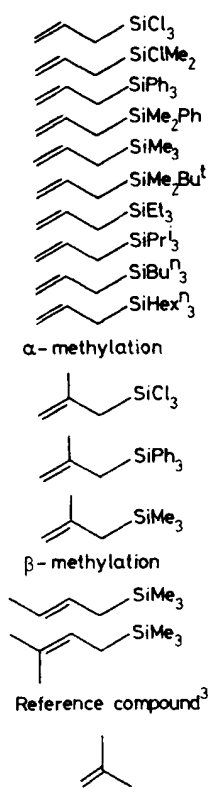

(1a) No reaction

(1b) $\quad 0.276$

(1c) $)^{\mathrm{a}} \quad 3.21$

(1d) $\quad 38.7$

(1e) 197

(1f) 204

(1g) 313

(1h) 439

(1i) 507

(1j) 542

(1k) 0.066

(11) $1.91 \times 10^{4}$

(Im) $>10^{5}$

(1n) $4.15 \times 10^{3}$

(1o) $1.56 \times 10^{3}$

23.8

a $(\mathbf{4 c}):(\mathbf{5 c})=1: 1.5$. 
We thank G. Horter for experimental assistance, and the Deutsche Forschungsgemeinschaft and the Fonds der Chemischen Industrie for financial support.

Received, 11th July 1988; Com. 8/02772B

\section{References}

1 E. W. Colvin, 'Silicon in Organic Synthesis,' Butterworths, London, 1981; W. P. Weber, 'Silicon Reagents for Organic Synthesis,' Springer, Berlin, 1983; I. Fleming, in 'Comprehensive Organic Chemistry,' eds. D. H. R. Barton and W. D. Ollis,
Pergamon Press, Oxford, 1979, vol. III, pp. 541; H. Sakurai, Pure Appl. Chem., 1982, 54, 1; D. Schinzer, Synthesis, 1988, 263; A. Hosomi, Acc. Chem. Res., 1988, 21, 200.

2 H. Mayr and R. Pock, Tetrahedron, 1986, 42, 4211.

3 R. Schneider, U. Grabis, and H. Mayr, Angew. Chem., Int. Ed. Engl., 1986, 25, 89; H. Mayr, R. Schneider, and C. Schade, Makromol. Chem. Macromol. Symp., 1988, 13/14, 43.

4 H. Mayr and R. Pock, Chem. Ber., 1986, 119, 2473.

5 R. Pock, H. Mayr, M. Rubow, and E. Wilhelm, J. Am. Chem. Soc., $1986,108,7767$.

6 P. B. D. de la Mare and R. Bolton, 'Electrophilic Additions to Unsaturated Systems,' Elsevier, Amsterdam, 1982; F. Freeman, Chem. Rev., 1975, 75, 439. 